\title{
Preventing carbon contamination of optical devices for $X$-rays: the effect of oxygen on photon-induced dissociation of $\mathrm{CO}$ on platinum
}

Article

Accepted Version

Risterucci, P., Held, G., Bendounan, A., Silly, M. G., Chauvet, C., Pierucci, D., Beaulieu, N. and Sirotti, F. (2012) Preventing carbon contamination of optical devices for X-rays: the effect of oxygen on photon-induced dissociation of $\mathrm{CO}$ on platinum. Journal of Synchrotron Radiation, 19 (4). pp. 570-573. ISSN 0909-0495 doi: https://doi.org/10.1107/S090904951202050X Available at https://centaur.reading.ac.uk/28844/

It is advisable to refer to the publisher's version if you intend to cite from the work. See Guidance on citing.

To link to this article DOI: http://dx.doi.org/10.1107/S090904951202050X

Publisher: International Union of Crystallography

All outputs in CentAUR are protected by Intellectual Property Rights law, including copyright law. Copyright and IPR is retained by the creators or other copyright holders. Terms and conditions for use of this material are defined in the End User Agreement. 


\section{www.reading.ac.uk/centaur}

\section{CentAUR}

Central Archive at the University of Reading

Reading's research outputs online 


\title{
Preventing carbon contamination of optical devices for X-rays: the effect of oxygen on photon-induced dissociation of $\mathrm{CO}$ on Platinum
}

\author{
Paul Risterucci, ${ }^{1}$ Geog Held, ${ }^{2}$ Azzedine Bendounan, ${ }^{1}$ Matthieu G. Silly, ${ }^{1}$ \\ Christian Chauvet, ${ }^{1}$ Debora Pierucci, ${ }^{1}$ Nathan Beaulieu, ${ }^{1}$ and Fausto Sirotti ${ }^{1}$ \\ ${ }^{1}$ Synchrotron SOLEIL, L'Orme des Merisiers, Saint-Aubin, BP 48, F-91192 Gif-sur-Yvette Cedex, France \\ ${ }^{2}$ Department of Chemistry, University of Reading, Whiteknights Reading RG6 6AD,UK
}

(Dated: May 1, 2012)

\begin{abstract}
Platinum is one of the most common coatings used to optimize mirrors reflectivity in soft X-ray beamlines. Normal operation results in optics contamination by carbon based molecules present in the residual vacuum of the beamlines. The reflectivity reduction induced by a carbon layer at the mirror surface is a major problem in synchrotron radiation sources. We present a time-dependent photoelectron spectroscopy study of the chemical reactions which take place at the $\operatorname{Pt}(111)$ surface under operating conditions. We show that the carbon contamination layer growth can be stopped and reversed by low partial pressures of oxygen for optics operated in intense photon beams at liquid nitrogen temperature. For mirrors operated at room temperature the carbon contamination observed for equivalent partial pressures of $\mathrm{CO}$ is reduced and effects of oxygen are observed on a long time scale.
\end{abstract}

\section{INTRODUCTION}

The interaction of carbon monoxide and oxygen on platinum surfaces is one of the most studied subjects in surface science. Due to their structural simplicity these molecules can be studied by a variety of experimental and theoretical techniques and their reaction mechanisms are well understood. On the other hand their interaction is of fundamental importance to key technologies, such as purification of hydrogen for fuel cells [1] or catalytic conversion of car exhaust gases [2]. CO oxidation has been widely studied at low- and ambient pressures [3-6]. The present study investigates the X-ray-induced surface chemistry of $\mathrm{CO}$ and $\mathrm{O}_{2}$ on the $\mathrm{Pt}(111)$ surface. It focuses on the kinetics of photon-induced dissociation of $\mathrm{CO}$ on $\mathrm{Pt}(111)$ and explores the possibility of removing the dissociation products by oxidation under the influence of soft X-rays. The experiments are strongly motivated by the desire to prevent carbon contamination of optical elements in soft X-ray and UV synchrotron beamlines, which are often coated with Pt in order to improve their reflectivity. Even with excellent base pressures in the $10^{-10}$ mbar range a significant increase in the partial pressures of carbon-containing molecules, mostly $\mathrm{CO}$, is observed in the mirror chambers of such beamlines when the optical elements are irradiated. Continuous operation in CO partial pressures results in thick carbon layers on the surfaces exposed to synchrotron radiation [7]. In the soft X-ray regime $(<1 \mathrm{keV})$, where the monochromators operate with high deviation angles, carbon contamination leads to sharp absorption features in the photon intensity vs energy and has dramatic effects on the photon polarization. This makes accurate X-ray absorption spectroscopy near the carbon K-edge difficult at best and often unreliable and prevents spectroscopic studies of many carbon-based molecules. In order to enable a comparison with realistic beamline conditions the experiments were carried out both with the sample at room temperature (RT) and cooled to liquid nitrogen (LN) temperatures (around $85 \mathrm{~K}$ ). The high power delivered by insertion devices at synchrotron radiation sources often makes it necessary to cool the first optical elements to cryogenic temperatures.

\section{EXPERIMENTAL DETAILS}

The measurements were performed taking advantage of the real time chemical reactivity set-up available on the experimental station of the TEMPO beamline at SOLEIL synchrotron [8]. The SCIENTA SES2002 electron energy analyzer and the beamline were operated at an overall resolution of about $25 \mathrm{meV}$ at photon energy $380 \mathrm{eV}$ and 50 $\mathrm{meV}$ for higher photon energy experiments [9]. The $\mathrm{Pt}(111)$ surface was cleaned by cycles of $\mathrm{Ar}^{+}$sputtering and annealing in oxygen pressure $\left(30 \mathrm{~min}\right.$ at $750 \mathrm{~K}$ with $\left.\mathrm{P}\left(\mathrm{O}_{2}\right)=1 \times 10^{-7} \mathrm{mbar}\right)$ followed by flashing to $1200 \mathrm{~K}$ for 1 min. High purity $\mathrm{CO}$ and $\mathrm{O}_{2}$ were introduced in the experimental chamber during data acquisition; their partial pressures were monitored with a mass spectrometer. The photon flux density on the sample was maximized to reach $10^{16} \mathrm{ph} \cdot \mathrm{s}^{-1} \cdot \mathrm{cm}^{-2}$, which is comparable to the photon flux density at beamline mirrors. With this photon density at the sample surface, the progress of $\mathrm{CO}$ adsorption and carbon contamination can be monitored by measuring the $\mathrm{C}-1$ s core level binding energies (BE) in a photoelectron spectroscopy experiment. 


\section{RESULTS AND DISCUSSION}

It is well-established that CO molecules can occupy two different adsorption sites on Pt(111), atop and a two-fold bridge site, in an upright geometry [10]. They are clearly identified by the C-1s binding energies of $286.8 \mathrm{eV}$ and $286.1 \mathrm{eV}$, respectively, as shown in the left panel of Fig. 1, which is in excellent agreement with literature [11, 12]. At the saturation coverage of $0.5 \mathrm{ML}$ the surface is covered by equal amounts of atop and bridge-bonded molecules in a very stable $\mathrm{c}(2 \times 4)$ overlayer structure $[11,12]$. The spectra presented in Fig. 1 were measured at RT after 5 minutes and after 5 hours of exposure to the soft X-ray beam. The measurements were performed with $380 \mathrm{eV}$ photon energy in ultra-high vacuum conditions after $\mathrm{CO}$ dosing for $10 \mathrm{~min}$ at $10^{-8}$ mbar to get the formation of the saturation coverage. Already 5 minutes later a $\mathrm{C}$-1s feature associated with dissociation can be detected in the $\mathrm{BE}$ region around $284 \mathrm{eV}$. Long exposure results in almost complete dissociation of the CO molecules. The two new peaks at $\mathrm{BE} 284.0$ and $283.6 \mathrm{eV}$ indicate the formation of a carbon layer with carbon atoms in two distinctly different chemical environments. These could be different adsorption sites on the Pt(111), e.g. hcp and fcc hollow sites or C-C bonded graphene-like vs isolated $\mathrm{C}$ atoms.

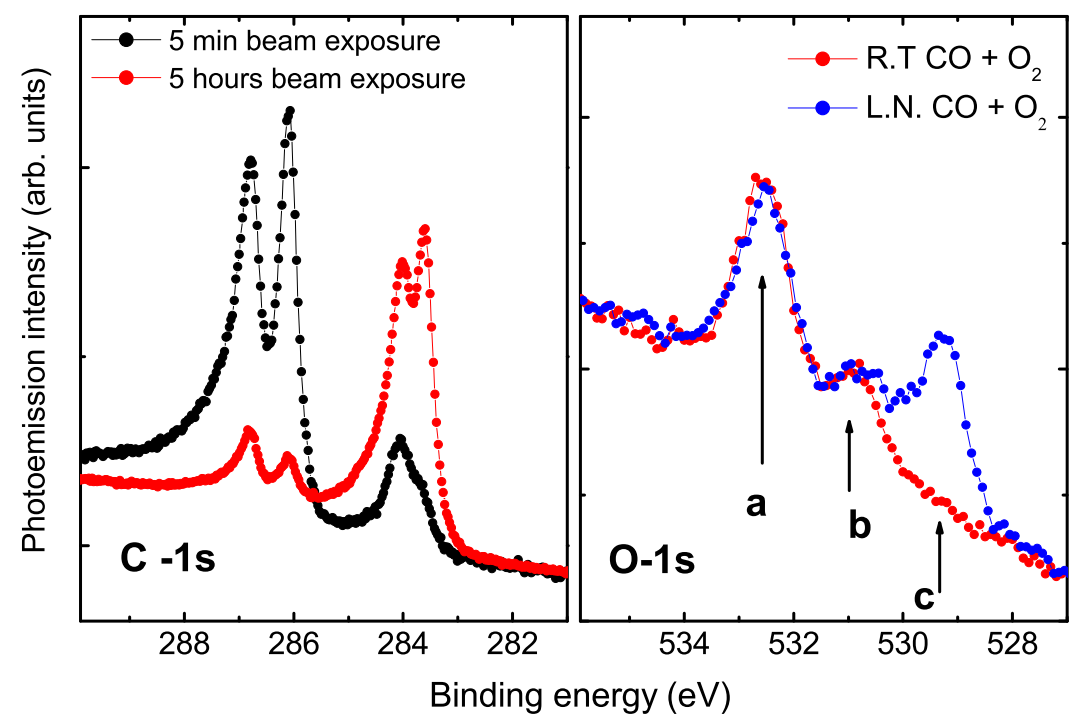

FIG. 1: Left: Carbon 1s photoemission spectra measured 5 minutes (black) and 5 hours (red) after CO dosing for 10 minutes in $10^{-8}$ mbar partial pressure at room temperature. The measurements were performed with 380 eV photon energy. The signatures of $C O$ adsorption sites are located at BE $286.8 \mathrm{eV}$ (atop) and $286.09 \mathrm{eV}$ (bridge). The carbon contamination is characterized by two peaks at BE $284.0 \mathrm{eV}$ and $283.6 \mathrm{eV}$. Right: Oxygen $1 \mathrm{~s}$ photoemission spectra measured with $630 \mathrm{eV}$ photon energy at room temperature (red) and at liquid nitrogen temperature (blue) after 10 min of simultaneous exposure at $\mathrm{CO}$ and $\mathrm{O}_{2}$ partial pressure of $0.9 \times 10^{-8}$ mbar and $4.9 \times 10^{-8}$ mbar, respectively. Peaks indicated by a and $b$ are assigned to the O-1s levels of the CO molecule on different sites on Pt(111), while peak $c$ is associated with the O-1s of the oxygen.

While the sticking probability of $\mathrm{CO}$ is almost independent of temperature between $85 \mathrm{~K}$ and RT, the adsorption of $\mathrm{O}_{2}$ is strongly temperature dependent. Below $96 \mathrm{~K}, \mathrm{O}_{2}$ is known to be physisorbed, at higher temperatures the molecules dissociate and chemisorb [13-17]. The physisorbed species is characterized by an O-1s BE of 535.0 eV whereas the chemisorbed atoms leads to a signal at BE $529.7 \mathrm{eV}$ [18]. Kim and co-workers showed that synchrotron radiation can dissociate $\mathrm{O}_{2}$ molecules at cryogenic temperatures [18].

As found by several previous studies, CO oxidation does not take place on Pt(111) below $120 \mathrm{~K}$ [19, 20], but when CO and $\mathrm{O}$ are co-adsorbed at higher temperatures the reaction probability is almost 1, following a Langmuir-Hinshelwood mechanism [20, 21]. The rate-limiting process at low temperature is the dissociation of $\mathrm{O}_{2}$. When irradiated with an intense soft X-ray beam, however, this dissociation can be photon-induced and the oxidation reactions $\mathrm{C}+\mathrm{O} \rightarrow \mathrm{CO}$ and $\mathrm{CO}+\mathrm{O} \rightarrow \mathrm{CO}_{2}$ can proceed.

The kinetics of photon-induced surface processes were monitored by measuring both C-1s and O-1s core level spectra with a photon energy of $630 \mathrm{eV}$ photon energy. This way, the chemical species (peak binding energy) as well as their coverages (peak area) can be recorded in real time during the experiment. In Fig. 2 we present the evolution of the signals related to $\mathrm{CO}$ and atomic carbon at RT and LN temperature over a period of 7 hours. The data points represent the integrated photoemission intensity after background subtraction for the BE regions 287.2 to $285.7 \mathrm{eV}$ (CO, filled circles) and 284.3 to $283.3 \mathrm{eV}$ (atomic carbon, open circles). The intensity axis is re-scaled such that the 
signal of the saturated layer at RT corresponds to $0.5 \mathrm{ML}$. The $\mathrm{CO}$ and $\mathrm{O}_{2}$ partial pressures are plotted on the top of Fig. 2.

At the beginning of the experiment, oxygen is introduced into the chamber at a pressure of $\mathrm{P}\left(\mathrm{O}_{2}\right)=4.9 \times 10^{-8}$ mbar. At $\mathrm{t}=0 \mathrm{~s}, 0.9 \times 10^{-8}$ mbar $\mathrm{CO}$ is added to the gas mixture and the evolution of the $\mathrm{C}-1 \mathrm{~s}$ signal is recorded over a period of three hours. On the time scale of Fig. 2 the surface is saturated by CO almost immediately at both temperatures. Under irradiation by the photon beam, the amount of intact CO on the surface then decreases at a longer time scale while the signal associated with contaminating carbon atoms increases.

The growth of the carbon layer is much faster at LN temperatures (blue symbols) than at RT (red symbols). The former can be described by $\Theta_{C}=\Theta_{\max }[1-\exp (-t / \tau)]$ with a characteristic time, $\tau$, of 65 min and a saturation coverage, $\Theta_{\max }$, of $0.75 \mathrm{ML}$. The O-1s spectra measured at low temperature (not shown here) indicate that around $1 \mathrm{ML}$ of physisorbed $\mathrm{O}_{2}$ molecules is present on the surface. Under the above conditions $\left(\mathrm{P}\left(\mathrm{O}_{2}\right)=4.9 \times 10^{-8} \mathrm{mbar}\right.$, $\mathrm{P}(\mathrm{CO})=0.9 \times 10^{-8}$ mbar, $\left.\mathrm{T} \approx 85 \mathrm{~K}\right)$, we observe that the carbon layer growth stops when a maximum coverage of 0.75 $\mathrm{ML}$ is reached. When the oxygen dosing is stopped (at $\mathrm{t}=160 \mathrm{~min}$ in Fig. 2) at low temperature the contamination signal starts to grow again, at a constant rate of $0.18 \mathrm{ML} / \mathrm{h}$, which does not seem to be affected even at the point where the $\mathrm{Pt}(111)$ surface is covered by a full monolayer of carbon atoms. This and the fact that a significant CO signal with the same BEs is still observed at this point indicates cluster-like 3D growth of the carbon contamination. In the right of Fig 2, we present the behavior observed if $\mathrm{O}_{2}$ is re-introduced in the chamber after a thick carbon layer has been grown. At low temperature this reduces the carbon contamination signal significantly with a characteristic time of $130 \mathrm{~min}$, compatible with reaching a sub monolayer coverage similar to the saturation coverage $\sim 0.75 \mathrm{ML}$ found in the first part of the experiment. In Fig. 2 one can observe that the thickness of the carbon contamination is lower than the one measured with lower photon energy without permanent CO dosing (presented in Fig. 1). The relative contributions of the photon flux, photon energy and gas pressure on the carbon contamination process goes

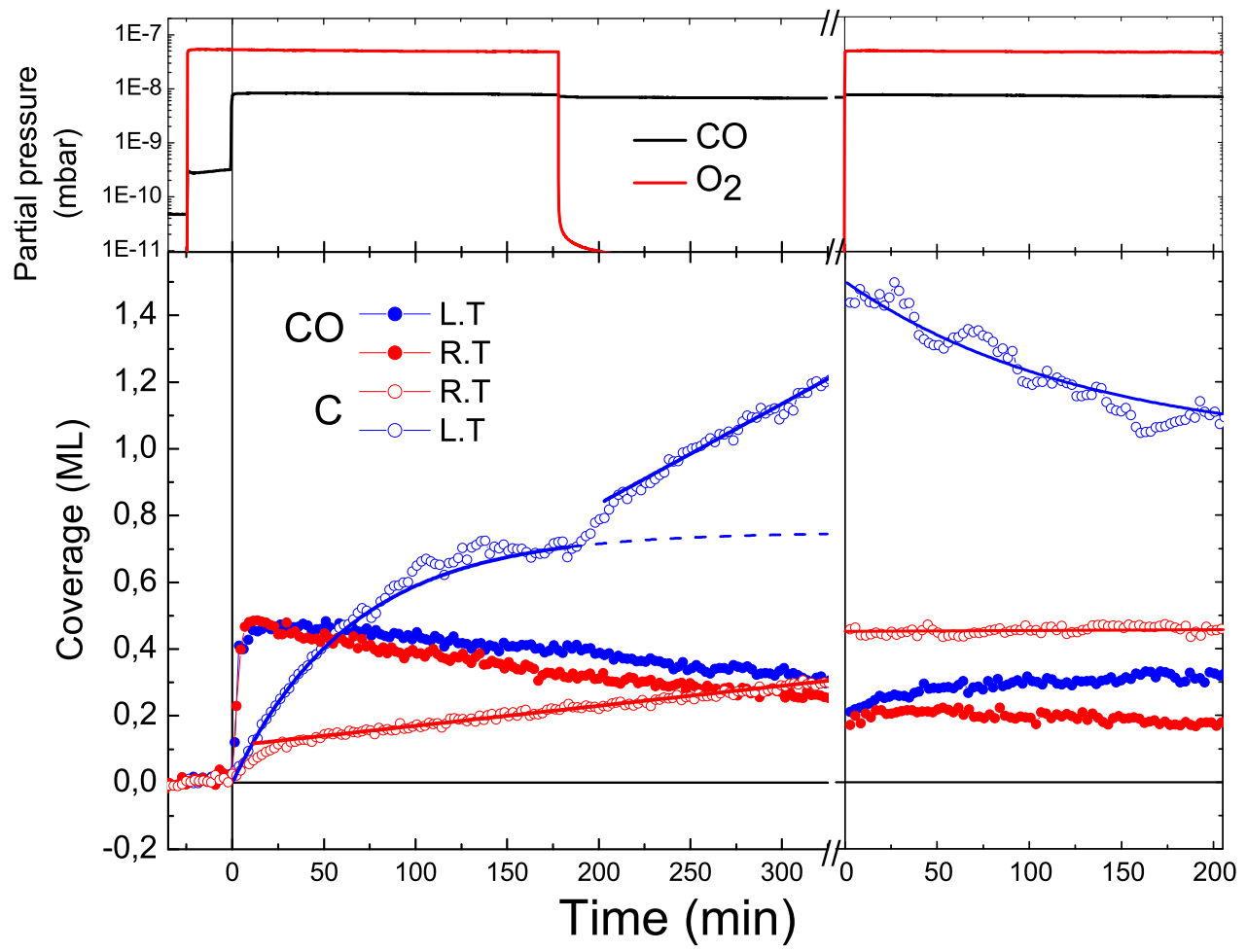

FIG. 2: Left panel: the lower part represents time evolution of the CO (filled symbols) and C (open symbols) XPS peak intensities (with photon energy $630 \mathrm{eV}$ ). The measured peak areas were normalized such that the CO saturation coverage at room temperature corresponds to $0.5 \mathrm{ML}$. The figure shows corresponding experiments at room temperature (red symbols) and at liquid nitrogen temperature (blue symbols). The upper part shows mass spectrometer measurements of the partial pressures at liquid nitrogen temperature. A CO partial pressure of $0.9 \times 10^{-8}$ mbar range is introduced in the chamber at $t=0$ s. 15 min after, $\mathrm{O}_{2}$ partial pressure of $4.9 \times 10^{-8}$ mbar has been introduced. In both experiments the surface is exposed to CO and $\mathrm{O}_{2}$ with a partial pressure ratio $\mathrm{P}\left(\mathrm{O}_{2}\right) / \mathrm{P}(\mathrm{CO})=5$. After 285 minutes the $\mathrm{O}_{2}$ partial pressure is switched off.

Right panel: contaminated surface resulting from the previous experiment are exposed again to a mixture of $\mathrm{CO}_{\text {and }} \mathrm{O}_{2}$ with a partial pressure ratio $\mathrm{P}\left(\mathrm{O}_{2}\right) / \mathrm{P}(\mathrm{CO})=5$. 
beyond the aim of the present work and must be studied in more details.

Although the signal of intact CO decreases at a similar rate for both temperatures, the increase in the contamination signal is slower at room temperature. After a steeper increase in the first $25 \mathrm{~min}$, the carbon coverage increases at a constant rate of $0.036 \mathrm{ML} / \mathrm{h}$ and reaches a stable saturation coverage of $0.45 \mathrm{ML}$ in presence of oxygen (right panel).

There is no change in the growth rate when oxygen is removed from the chamber; the build-up of the carbon layer at room temperature is not affected by the presence of $\mathrm{O}_{2}$. Since the carbon contamination process is slower at room temperature, the effect of the oxygen exposure requires much longer experimental sessions to obtain a better description of the phenomenon taking place on the real optical elements of the beamline.

The faster build-up of atomic carbon at low temperatures is most likely related to the fact that the oxidative removal of atomic carbon, $\mathrm{C}+\mathrm{O} \rightarrow \mathrm{CO}$, is thermally activated. The rate-limiting step is $\mathrm{O}_{2}$ dissociation. Therefore, if atomic oxygen can be supplied by photo-dissociation of $\mathrm{O}_{2}$ the reaction can still proceed even at low temperature. The confirmation comes from the comparison of the O-1s core levels measured at low temperature and at room temperature after 10 minutes of $\mathrm{O}_{2}$ and $\mathrm{CO}$ exposure. (see right panel of Fig. 1). At this stage of the reaction $\mathrm{CO}$ molecules are still present on the surface as indicated by the a) and b ) O-1s peaks at 532.5 and $531 \mathrm{eV}$ but at cryogenic temperature activated oxygen is also observed on the surface (peak at $529.3 \mathrm{eV}$ binding energy). O-1s spectra measured as a function of time shows that a constant amount of $\mathrm{O}_{2}$ does adsorb on the surface also when atomic carbon is present. Note that the presence of this oxygen amount on the optical surface induces a reduction of photon flux at the oxygen K-edge of about $10 \%$. The rate of oxidative removal of carbon increases with the amount of carbon on the surface until an equilibrium is reached. The same mechanism also explains the removal of the thick carbon layer after the oxygen supply had been interrupted (Fig. 2). From the kinetic constants determined in our experiment we estimate the photo-dissociation cross section for $\mathrm{CO}$ as $10^{-19} \mathrm{~cm}^{2}$; the photon-induced oxidation has a rate of around $10^{-4} \mathrm{ML} . \mathrm{s}^{-1}$. At RT thermally activated oxygen is not available on the surface. The oxidation of carbon runs in parallel with $\mathrm{CO}$ oxidation, $\mathrm{CO}+\mathrm{O} \rightarrow \mathrm{CO}_{2}$, and balances the photon-induced dissociation of $\mathrm{CO}$.

\section{CONCLUSION}

In summary, we have reproduced the carbon contamination conditions of Pt coated mirrors in a surface science experiment. We have identified the binding energies of the carbon contaminating species and followed in real time the chemical reactions taking place at the Pt surface at RT and at LN temperature. For mirrors kept at LN temperature, the presence of a relatively low $\mathrm{O}_{2}$ partial pressure in the $10^{-8}$ mbar range in the chamber prevents the carbon contamination growth and reduces a thick carbon layer in the submonolayer range. Our findings are confirmed by the operation of the TEMPO beamline under real conditions, where the same partial pressures described above are now applied in our mirror chambers hosting liquid nitrogen cooled mirrors. In the last year of operation, we have not observed significant carbon contamination in contrast to the experience before when no oxygen was introduced. For mirrors operated at RT and in the presence of oxygen partial pressure, the carbon contamination layer appears to be saturated at only 0.45 ML. Such an observation needs to be more explored with much longer experimental sessions.

[1] : B.C.H. Steele and A. Heinzel, Nature 414, 345 (2001).

[2] : X. Xie, Y.Li, Z.-Q. Liu, M. Haruta, W. Shen, Nature 458, 746 (2009).

[3] : G. Ertl, Surf. Sci. 299-300, 742 (1994).

[4] : J. Wintterlin, S. Völkening, T.V.W. Janssens, T. Zambelli, G. Ertl, Science 278, 1931 (1997).

[5] : F. Gao, Y. Wang, Y. Cai, and D. W. Goodman, J. Phys. Chem. C 113, 174 (2009).

[6] : B. L. M. Hendriksen and J.W.M. Frenken, Phys. Rev. Lett. 89, 046101 (2002).

[7] : C. Chauvet, F. Polack, M.G. Silly, B. Lagarde, M. Thomasset, S. Kubsky, J.P. Duval, P. Risterucci, B. Pilette, I. Yao, N. Bergeard, F. Sirotti, J. Sync. Rad. 18, 761 (2011).

[8] : F. Polack, M.G. Silly, C. Chauvet, B. Lagarde, N. Bergeard, M. Izquierdo, O. Chubar, D. Krizmancic, M. Ribbens, J.P. Duval, C. Basset, S. Kubsky, F. Sirotti, AIP Conf. Proc. 1234, 185 (2010).

[9] : N. Bergeard, M.G. Silly, D. Krizmancic, C. Chauvet, M. Guzzo, J.P. Ricaud, M. Izquierdo, L. Stebel, P. Pittana, S. Sergo, G. Cautero, G. Dufour, F. Rochet, F. Sirotti, J. Sync. Rad. 18, 245 (2011).

[10] : H. Froitzheim, H. Hopster, H. Ibach, S. Lehwald, Appl. Phys. 13, 147 (1977).

[11] : M. Kinne, T. Furhmann, C.M. Whelan, J.F Zhu, J. Pantförder, M. Probst, G. Held, R. Denecke, H.P. Steinrück, J. Chem. Phys. 117, 10852 (2002).

[12] : F. Bondino, G. Comelli, F. Esch, A. Locatelli, A. Baraldi, S. Lizzit, G. Paolucci, R. Rosei, Surf. Sci. 459, 467 (2000).

[13] : J.L. Gland, B.A. Sexton, G.B. Fisher, Surf. Sci. 95, 587 (1980).

[14] : C. Puglia, A. Nilsson, B. Hernnäs, O. Karis, P. Bennich, N. Martensson, Surf. Sci. 342, 119 (1995).

[15] : P.D. Nolan, B.R. Lutz, P.L. Tanaka, J.E. Davis, C.B. Mullins, J. Chem. Phys. 111, 3696 (1999). 
[16] : K. Gustafsson, S. Andersson, J. Chem. Phys. 120, 7750 (2004).

[17] : B.C. Stipe, M.A. Rezaei, W. Ho, J. Chem. Phys. 107, 6443 (1997).

[18] : Y.S. Kim, A. Bostwick, E. Rotenberg, P.N. Ross, S.C. Hong, B.S. Mun, J. Chem. Phys. 133, 034501 (2010).

[19] : M. Kinne, T. Fuhrmann, J.F. Zhu, C.M. Whelan, R. Denecke, H.P. Steinrück, J. Chem. Phys. 120, 7113 (2004).

[20] : K.H. Allers, H. Pfnür, P. Feulner, D. Menzel, J. Chem. Phys. 100, 3985 (1994).

[21] : C.T. Campbell, G. Ertl, H. Kuipers, J. Segner, J. Chem. Phys. 73, 5862 (1980). 